Check for updates

Cite this: RSC Adv., 2017, 7, 29306

Received 27th March 2017

Accepted 21st May 2017

DOI: $10.1039 / \mathrm{c} 7 \mathrm{ra03549g}$

rsc.li/rsc-advances

\section{Double-layer core/shell-structured nanoparticles in polyarylene ether nitrile-based nanocomposites as flexible dielectric materials $\uparrow$}

\author{
Yong You, Weihua Han, Ling Tu, Yajie Wang, Renbo Wei (D) and Xiaobo Liu* \\ Copper tetra-amine phthalocyanine $\left(\mathrm{NH}_{2}-\mathrm{CuPC}\right)$ was grafted onto barium titanate $\left(\mathrm{BaTiO}_{3}\right)$ whose surface \\ was modified by carboxyl-functionalized polyarylene ether nitrile (CPEN), (CPEN-f-BT@CuPc), using rotary \\ coating technology combined with an ultrasonic dispersion technology and a post-treatment bonding \\ process. The CPEN-f-BTACuPc/polyarylene ether nitrile (PEN) nanocomposites show stable dielectric \\ constant and energy density in the temperature range of $25-160{ }^{\circ} \mathrm{C}$, which demonstrate huge potential \\ for use as organic film capacitors.
}

\section{Introduction}

With the rapid development of the electric industry, lightweight and high-energy-density materials have attracted significant attention owing to their potential application in capacitors and batteries. ${ }^{1,2}$ Capacitor films with excellent dielectric properties and thermal stability under demanding conditions play an important role in various applications such as in electronic systems. However, pure polymers suffer from the limitation of low dielectric constant. Therefore, preparing polymer-based composites, which combine the advantages of polymers and inorganic nanofillers, is one of the effective ways to solve this problem..$^{3-6}$

Polyarylene ether nitriles (PENs), as special thermoplastic engineering materials, are well-known for their outstanding properties, including excellent mechanical properties and chemical inertia, radiation resistance, and high thermal stability, ${ }^{7-10}$ and have been used in automotive and outer space fields that encounter high temperature or high radiation exposure. In addition, nitrile groups with strong polarity on the side of an aromatic ring can effectively promote the adhesion of the macromolecular main-chain to many substrates. ${ }^{11}$ Moreover, high solubility of PEN in polar organic solvents, including $N$ methyl-2-pyrrolidinone (NMP) and $N, N$-dimethylformamide (DMF), and its wide range of processing window make this polymer to be readily processed into different-shaped prod-

Research Branch of Advanced Functional Materials, School of Microelectronics and Solid-State Electronics, High Temperature Resistant Polymer and Composites Key Laboratory of Sichuan Province, University of Electronic Science and Technology of China, Chengdu, 610054, P. R. China.E-mail: weirb10@uestc.edu.cn; liuxb@uestc. edu.cn; Fax: +86-28-83207326; Tel: +86-28-83207326

$\dagger$ Electronic supplementary information (ESI) available. See DOI: 10.1039/c7ra03549g ucts. ${ }^{12}$ Apart from the polymers, proper selection of fillers of different types and shapes also helps in improving the electrical properties of the polymer nanocomposites. Recently, high dielectric constant ceramic-based polymer nanocomposites have attracted significant interest. These materials generally show relatively low dielectric loss because of the dielectric character of the polymer and the ceramic particles..$^{13}$ Barium titanate $\left(\mathrm{BaTiO}_{3}\right)$, as one of the typical ceramic nanoparticles, is well-known for its outstanding properties such as stable chemical structure, relatively high dielectric constant, and low dielectric loss, ${ }^{14-18}$ and one of the most common additives used to reinforce the performance of PEN. ${ }^{19}$ These ceramics behave as insulators as they have high band gap such that the accumulation of charges takes place only on the application of an electric field.

Although it is extremely important, it is quite a challenge to homogeneously disperse the fillers in PEN to achieve the aforementioned excellent properties. It has been found that the nanoparticle surface chemistry as well as the composite microstructure are among the most important factors determining the dielectric properties of the ceramic nanoparticlebased polymer composites. ${ }^{20,21}$ Therefore, it is a prerequisite to improve the performances of the PEN-based composites using effective techniques, such as surface modification, chemical grafting, and physical blending, such that they can be used under some critical conditions..$^{22-25}$

In this study, the surface of $\mathrm{BaTiO}_{3}$ was modified with CPEN and $\mathrm{NH}_{2}$-CuPc, and the obtained novel core-shell nanoparticle (CPEN-f-BT@CuPc) was used to reinforce the performance of biphenyl polyarylene ether nitrile (PEN). The PEN-based composite films can be used as dielectric materials with broad operating temperature and high thermal stability. The dielectric properties and energy density characteristics at high temperatures have been studied in detail. 


\section{Experimental}

\section{Materials}

$\mathrm{N}$-Methyl-2-pyrrolidone (NMP; purity 99\%), $\mathrm{N}, \mathrm{N}$-dimethylformamide (DMF; purity 99\%), N,N-dimethylacetamide (DMAc; purity 99\%), and tetrahydrofuran (THF) were purchased from Tianjin Bodi Chemical Holding Co. Ltd, Tianjin, China. $\mathrm{BaTiO}_{3}$ (average diameter $=60 \mathrm{~nm}$ ) was purchased from TPL Co. Ltd, USA. Potassium carbonate $\left(\mathrm{K}_{2} \mathrm{CO}_{3} ; \mathrm{AR}\right)$, toluene (AR), acetone (AR), alcohol (AR), biphenol (BP), phenolphthalein (PPL), 2,6-dichlorobenzonitrile (DCBN), 4-nitrophthalonitrile, $\mathrm{Na}_{2} \mathrm{~S} \cdot 9 \mathrm{H}_{2} \mathrm{O}, \quad \mathrm{Cu}(\mathrm{AC})_{2} \cdot \mathrm{H}_{2} \mathrm{O}$, and hydrochloric acid were purchased from Chengdu Haihong Chemicals (Chengdu, China). All other reagents were commercially available products and used as received without further purification. CPEN and PEN were synthesized via nucleophilic aromatic substitution polymerization using a previously reported method, ${ }^{26,27}$ and the corresponding synthetic routes of PEN are shown in Fig. S1. $\dagger$ $\mathrm{NH}_{2}$-CuPc was synthesized using a previously reported protocol. ${ }^{28}$ The details of the synthesis of PEN, CPEN, and $\mathrm{NH}_{2}$ CuPc are shown in the ESI. $\dagger$

\section{Surface functionalization of the $\mathrm{BaTiO}_{3}$ nanoparticles}

The $\mathrm{BaTiO}_{3}$ nanoparticles were surface-functionalized with CPEN (CPEN-f-BT) via rotary coating technology according to a previous report, ${ }^{29}$ and the corresponding route is shown in Fig. 1a. $\mathrm{BaTiO}_{3}$ (BT, average diameter $=60 \mathrm{~nm}, 10.0 \mathrm{~g}$ ) in 100 $\mathrm{mL}$ of tetrahydrofuran (THF) was ultrasonicated for $2 \mathrm{~h}$ to form a homogeneous suspension. CPEN ( $4.0 \mathrm{~g}$ ) in $40 \mathrm{~mL}$ of THF was slowly added into the suspension. The mixture was stirred using ultrasonication for $2 \mathrm{~h}$. After evaporation of the solvent, the particles were heated to $200{ }^{\circ} \mathrm{C}$ and kept at this temperature for 4 h. CPEN-functionalized $\mathrm{BaTiO}_{3}$ (CPEN-f-BT) nanoparticles were obtained by washing three times each with THF, ethanol, and deionized water. Then, CPEN-f-BT (2.0 g), $\mathrm{NH}_{2}$-CuPc (0.5 g), and DMAc $(20 \mathrm{~mL})$ were stirred at $160{ }^{\circ} \mathrm{C}$ for $4 \mathrm{~h}$ to form a homogeneous suspension. The surface-grafted particles were post-treated via amidation at $200{ }^{\circ} \mathrm{C}$ for $20 \mathrm{~h}$; the CuPc particles linked with PEN via chemical and hydrogen bonds. ${ }^{11}$ The CPENf-BT@CuPc core-shell nanoparticles were obtained by washing three times each with DMAc, ethanol, and deionized water.

\section{Preparation of the CPEN-f-BT@CuPc/PEN films}

CPEN-f-BT@CuPc/PEN multicomponent nanocomposite films were obtained via a solution casting method according to a previous report. ${ }^{22}$ CPEN-f-BT@CuPc/PEN nanocomposites (0 wt $\%, 2.0 \mathrm{wt} \%, 5.0 \mathrm{wt} \%, 10.0 \mathrm{wt} \%$, and $20.0 \mathrm{wt} \%$ ) were homogeneously suspended in NMP by stirring and ultrasonicating for $2 \mathrm{~h}$. Then, the mixture was cast on a clean horizontal glass plate and then dried in an oven at $80,100,120,160$, and $200{ }^{\circ} \mathrm{C}(1 \mathrm{~h}$ each) to completely remove the solvent. After naturally cooling down to room temperature, the composite films with a thickness of about $60 \mu \mathrm{m}$ were obtained.

\section{Characterization}

Transmission electron microscopy (TEM) images were obtained using a JEM-2100F electron microscope with an accelerating voltage of $200 \mathrm{kV}$. Scanning electron microscopy (SEM) and energy dispersive spectrometry (EDS) were carried out using a JEOL JSM-5900LV at $20 \mathrm{kV}$. SEM samples were fractured in liquid nitrogen and then sputtered with gold on the fractured

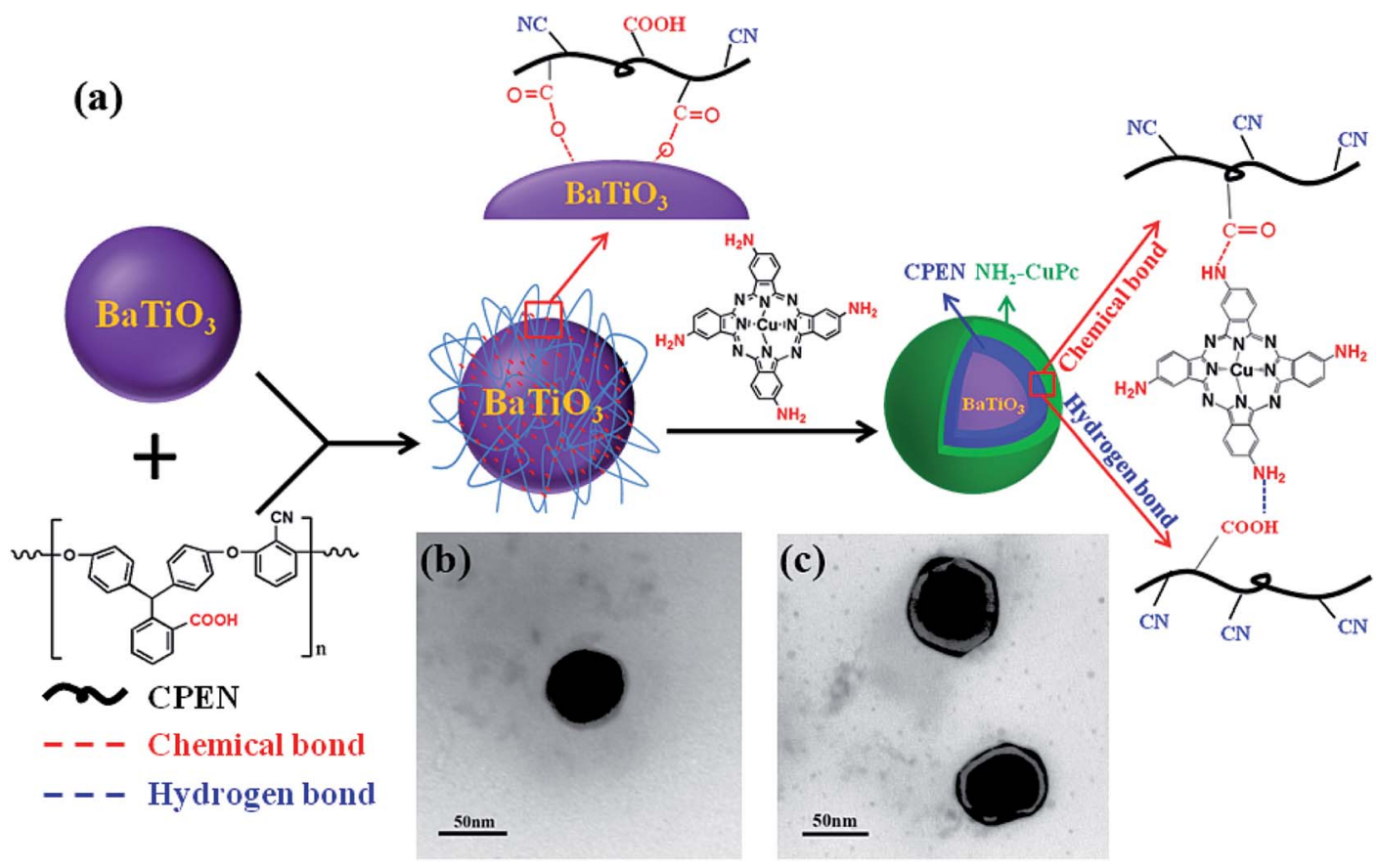

Fig. 1 The preparation and characterization of the nanoparticles: (a) route of preparation; (b) and (c) TEM images of CPEN-f-BT and CPEN-fBT@CuPc, respectively. 
surface. X-ray photoelectron spectroscopic (XPS) measurements were carried out via an ESCA 2000 (VG Microtech, UK) using a monochromic $\mathrm{Al} \mathrm{K} \alpha(h \nu=1486.6 \mathrm{eV}) \mathrm{X}$-ray source. Fourier transform infrared (FTIR) measurements were carried out via a Shimadzu 8400S FTIR spectrophotometer by incorporating the samples in the $\mathrm{KBr}$ pellets. Ultraviolet-visible (UV-vis) absorption spectra were obtained using a TU1800 ultravioletvisible spectrophotometer (Beijing Purkinje General Instrument Co., Ltd). X-ray diffraction (XRD) analysis was carried out using a Rigaku RINT2400 X-ray diffractometer with $\mathrm{Cu} \mathrm{K} \alpha$ radiation. The dielectric properties of the samples were tested using a TH 2819A precision LCR meter (Tong hui Electronic Co., Ltd.). The electric breakdown strength was tested by a Dielectric Withstand Voltage Tester (ZJC-50 kV).

Differential scanning calorimetry (DSC) analysis was conducted using a TA Instruments DSC Q100 (NewCastle, DE, USA) modulated thermal analyzer. To eliminate the residual thermal history, two scanning cycles of heating and cooling were performed for each sample in the temperature range from 50 to $300{ }^{\circ} \mathrm{C}$ at a heating rate of $10{ }^{\circ} \mathrm{C} \mathrm{min}^{-1}$ under a nitrogen atmosphere. The second heating scan was used to determine the $T_{\mathrm{g}}$ of the corresponding samples. Thermal gravimetric analysis (TGA) was carried out using a TA Q50 system under a nitrogen atmosphere at a heating rate of $20{ }^{\circ} \mathrm{C} \mathrm{min}^{-1}$. Mechanical properties of the samples were tested using a SANS CMT6104 series desktop electromechanical universal testing machine (Shenzhen, China).

\section{Results and discussions}

The TEM observations are the intuitionistic characterization of the surface of $\mathrm{BaTiO}_{3}$ with CPEN and CPEN-f-BT functionalized with CuPc. As shown in Fig. 1b and c, it can be intuitively seen that the polymer layer is coated on the surface of $\mathrm{BaTiO}_{3}$ (Fig. 1b), and the outermost black layer belongs to CuPc (Fig. 1c), which has been successfully grafted onto the surface of CPEN-f-BT.

As shown in Fig. 2a, it can be clearly seen that the UV-vis curve of CPEN-f-BT@CuPc has two strong absorption bands (Q-band: 600-700 nm and B-band: 300-400 nm) in both the visible and ultraviolet regions as compared to that of CPEN-f-BT, which is attributed to the $\pi-\pi^{*}$ transition from the phthalocyanine rings and the B-band absorption of the phthalocyanine ring. ${ }^{30}$ Wideangle X-ray (WAXD) was further employed to confirm the surface of CPEN-f-BT functionalized with CuPc, as shown in Fig. 2b. Compared to the pattern of $\mathrm{BaTiO}_{3}$, all the peaks of $\mathrm{BaTiO}_{3}$ are reserved and three extra obvious diffraction peaks at $14.2^{\circ}, 24.6^{\circ}$, and $28.9^{\circ}$ can be observed in the pattern of CPEN-fBT@CuPc, which are the main peaks of CuPc. All these results clearly reveal that CuPc coexists with the CPEN-f-BT nanoparticles. Fig. $2 \mathrm{c}$ is the SEM image that shows the uniform CPEN-fBT@CuPc core-shell nanoparticles. Moreover, the surface chemical composition of the CPEN-f-BT@CuPc core-shell nanoparticles was further determined by EDS measurements, as described in Fig. 2d. The EDS pattern of the nanoparticle demonstrates that the CPEN-f-BT@CuPc core-shell nanoparticles are mainly composed of barium (Ba), titanium (Ti), oxygen (O), carbon $(\mathrm{C})$, nitrogen $(\mathrm{N})$, and copper $(\mathrm{Cu})$ elements, which proves the existence of $\mathrm{BaTiO}_{3}$ and CuPc. The XPS provides essential and useful information about the chemical bond energy, which represents different kinds of chemical bonds, of a particular element. As shown in Fig. S2, $\uparrow$ the full scanned XPS spectrum demonstrated the existence of barium (Ba), titanium (Ti), oxygen $(\mathrm{O})$, carbon $(\mathrm{C})$, nitrogen $(\mathrm{N})$, and copper $(\mathrm{Cu})$ elements in the CPEN-f-BT@CuPc core-shell nanoparticles. The N1s spectrum of CPEN-f-BT@CuPc could be quantitatively differentiated into the nitrogen species of $\mathrm{C}-\mathrm{N}(398.13 \mathrm{eV}), \mathrm{C} \equiv \mathrm{N}(398.33 \mathrm{eV}),-\mathrm{NH}_{2}$ (399.35 eV), $-\mathrm{CONH}(399.88 \mathrm{eV})$, and $\mathrm{C}=\mathrm{N}\left(400.54 \mathrm{eV}\right.$ ) (Fig. 2e)..$^{\mathbf{3 1 , 3 2}}$ The nitrogen species of $\mathrm{C}-\mathrm{N}$ and $\mathrm{C}=\mathrm{N}$ originated from the
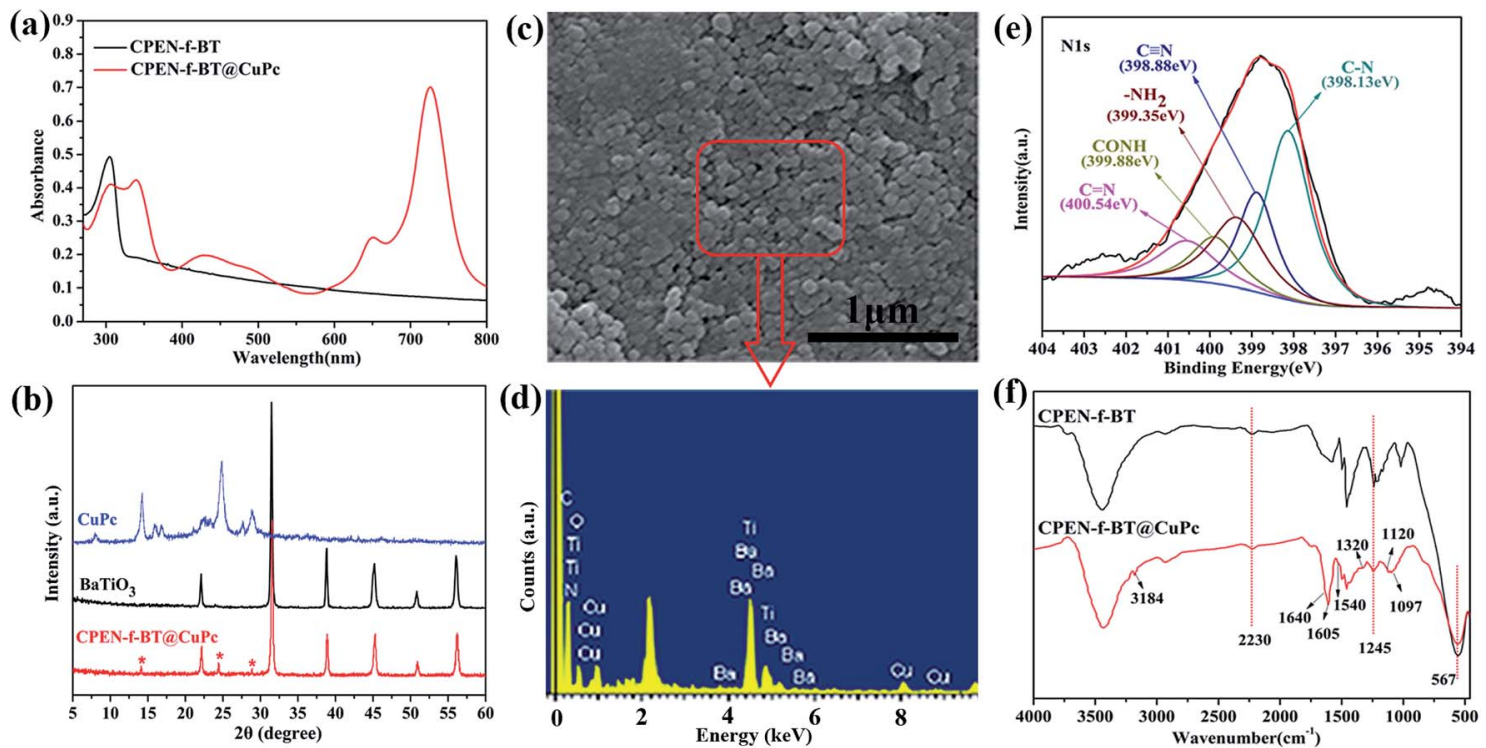

Fig. 2 The characterization of nanoparticles: (a) UV-vis spectrum; (b) XRD patterns; (c) SEM image; (d) EDS patterns; (e) XPS N1s core-level spectrum; and (f) FTIR spectrum. 
phthalocyanine ring, and $\mathrm{C} \equiv \mathrm{N}$ originated from the cyano groups on CPEN. $-\mathrm{NH}_{2}$ belongs to the amino group on $\mathrm{NH}_{2}-\mathrm{CuPc}$, which is not involved in the chemical reaction. The peak at $399.88 \mathrm{eV}$ is attributed to the amide bonds, ${ }^{31}$ which are formed by the reaction between $-\mathrm{COOH}$ from $\mathrm{CPEN}$ and $-\mathrm{NH}_{2}$ from $\mathrm{NH}_{2}-\mathrm{CuPc}$ in the system. The FTIR spectra of CPEN-f-BT and CPEN-f-BT@CuPc are shown in Fig. 2f. The absorption band at $2230 \mathrm{~cm}^{-1}$ corresponds to the symmetrical stretching vibration of the nitrile groups $(-\mathrm{CN}),{ }^{10}$ and the characteristic band at $1245 \mathrm{~cm}^{-1}$ belongs to aryl ether. ${ }^{33}$ Moreover, the strong band at $567 \mathrm{~cm}^{-1}$ originated from the Ti-O vibration. ${ }^{34}$ Furthermore, the characteristic absorption peaks at $1097 \mathrm{~cm}^{-1}, 1120 \mathrm{~cm}^{-1}$, and $1605 \mathrm{~cm}^{-1}$ in the spectrum of CPEN-f-BT@CuPc belong to the phthalocyanine ring, and the weak band at $3184 \mathrm{~cm}^{-1}$ corresponds to the characteristic absorption of the $-\mathrm{NH}$ stretching vibrations. Moreover, the enlarged FTIR spectrum from 1200 to 2000 wavenumber is shown in Fig. S3. $\dagger$ Small peaks at 1640,1540 , and $1320 \mathrm{~cm}^{-1}$ can be observed in this enlarged FTIR spectrum, which also confirm the formation of amide bonds. The low intensity of these peaks might result from the low content of the amide bonds.

In addition, the thermal and mechanical properties of the PEN-based nanocomposites are shown in Fig. 3. It can be seen that the glass transition temperatures $T_{\mathrm{g}}$ of the nanocomposites with different contents of CPEN-f-BT@CuPc are around $217{ }^{\circ} \mathrm{C}$ (Fig. 3a). The TGA curves of the nanocomposites are shown in Fig. S4; $\dagger$ all samples show excellent thermal resistance with an initial decomposition temperature $\left(T_{5 \%}\right)$ of over $527{ }^{\circ} \mathrm{C}$ in a $\mathrm{N}_{2}$ atmosphere. Fig. $3 \mathrm{~b}$ shows the tensile strength and tensile modulus of the nanocomposites. The tensile strength and modulus of samples increase from 116.5 and $1943 \mathrm{MPa}$ to 121.4 and $2192 \mathrm{MPa}$, respectively, when the content of nanofillers increases from 0 to $2.0 \mathrm{wt} \%$. As more nanofillers are incorporated, the tensile strength and modulus of nanocomposite films decrease with the increasing content of nanofillers. This results from the fact that a large number of CPEN-f-BT@CuPc tend to agglomerate, which decreases the tensile strength of the nanocomposites. The corresponding cross-sectional micromorphology of the composite films is shown in Fig. S5. $\dagger$ However, the tensile strength and modulus are higher than $80 \mathrm{MPa}$ and $1600 \mathrm{MPa}$, respectively, even when the content of nanofillers increases to $20.0 \mathrm{wt} \%$.

In addition, the elongation at break of all the samples is over 11.6\% (as shown in Fig. S6 $\dagger$ ), meaning that these materials can be used as flexible dielectric materials. These results indicate that the nanocomposites show excellent mechanical and thermal properties.
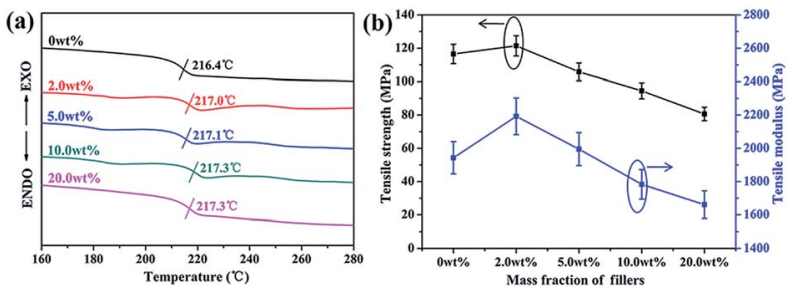

Fig. 3 DSC curves (a) and mechanical properties (b) of the CPEN-fBTaCuPc/PEN composite films.
Furthermore, the dielectric permittivity and loss tangent of the nanocomposites were measured as a function of frequency ranging from $100 \mathrm{~Hz}$ to $200 \mathrm{kHz}$ at room temperature. As shown in Fig. 4a, the dielectric constant of all the nanocomposite films shows a weak decrement with the increasing frequency, which indicates that the samples have good permittivity-frequency stability. The dielectric constant of the PEN-based films increases from 3.9 to 9.0 at $1 \mathrm{kHz}$ with the content of CPEN-fBT@CuPc increasing from $0 \%$ to $20.0 \mathrm{wt} \%$. This is because the polymer-based nanocomposite can form micro-capacitor networks, which has been reported elsewhere. ${ }^{35}$

Furthermore, the number of micro-capacitors increases with the increasing content of the nanofillers, which improves the dielectric constant of the CPEN-f-BT@CuPc/PEN composite films. Compared to that of the $\mathrm{BaTiO}_{3} / \mathrm{PEN}$ composite films, the dielectric constant of the CPEN-f-BT@CuPc/PEN composite films is much higher at the same content of the nanofillers. ${ }^{36}$ This is mainly due to the fact that the surface-functionalized $\mathrm{BaTiO}_{3}$ nanoparticles have good compatibility with the polymer matrix, which provides better dispersion in the nanocomposite films (as shown in Fig. S5†). Moreover, the phthalocyanine ring and strong polar nitrile groups on the surface of $\mathrm{BaTiO}_{3}$ can also increase the electric charge. In addition, good compatibility of the nanoparticles within the polymer matrix also contributes to low loss tangent. As can be seen from Fig. $4 \mathrm{~b}$, the dielectric loss of the nanocomposite films at $1 \mathrm{kHz}$ increases from 0.0115 to 0.0312 when the nanofillers content increases. The breakdown strength of the nanocomposite films was tested at room temperature, and the results are shown in Fig. 4c. It can be seen that the breakdown strength of the samples decreases from 202.8 to $168.6 \mathrm{kV} \mathrm{mm}^{-1}$ as the nanofillers content increases. The energy density was calculated from eqn (1) and the results are presented in Fig. 4d. The energy density $(U)$ of a capacitor is given by the following equation:

$$
U=\frac{1}{2} \varepsilon_{0} \varepsilon_{\mathrm{r}} E_{\mathrm{b}}^{2}
$$
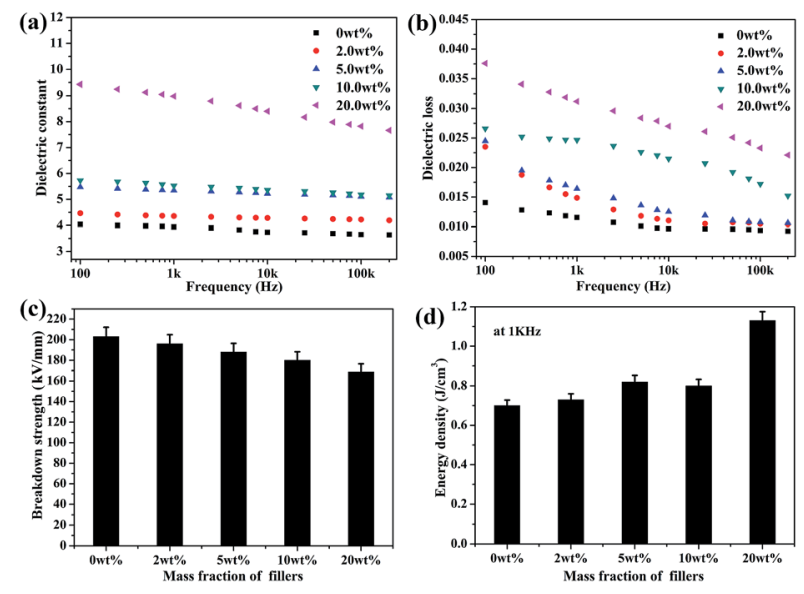

Fig. 4 The dielectric properties (a), dielectric loss (b), breakdown strength (c), and energy density at $1 \mathrm{kHz}$ (d) of the CPEN-f-BTaCuPc/ PEN composite films. 

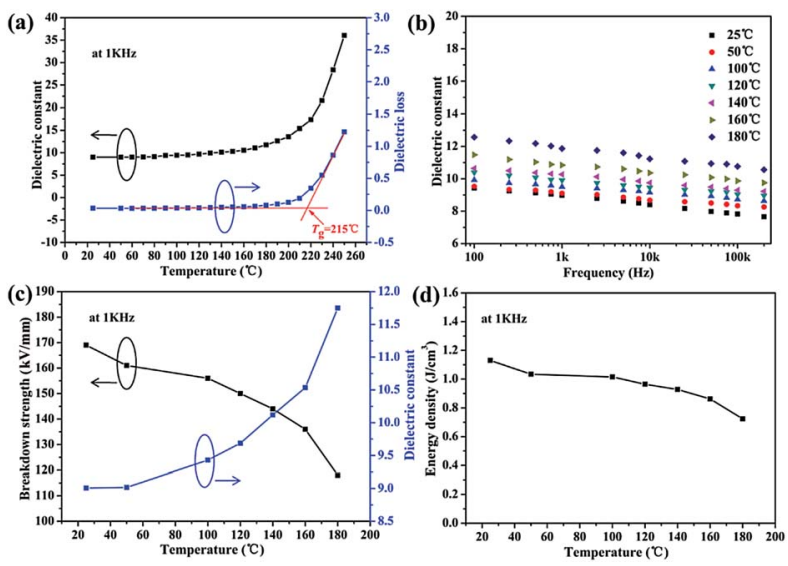

Fig. 5 The energy storage characteristics of the CPEN-f-BTaCuPc/ PEN composite films: (a), (c), and (d) the dielectric constant, breakdown strength, and energy density at $1 \mathrm{kHz}$, respectively. (b) The dielectric constant at different temperatures.

where $\varepsilon_{0}$ is the vacuum permittivity, $\varepsilon_{\mathrm{r}}$ is the permittivity of the composites, and $E_{\mathrm{b}}$ is the breakdown strength. Combined with $\varepsilon_{\mathrm{r}}, E_{\mathrm{b}}$, and eqn (1), the calculated results indicate that when the content of the nanofillers reaches $20.0 \mathrm{wt} \%, U$ increases by $61 \%$, from 0.70 to $1.13 \mathrm{~J} \mathrm{~cm}^{-3}$. This is the result of a large increase in $\varepsilon_{\mathrm{r}}$ and a small decrease in $E_{\mathrm{b}}$, which is also contributed to the fact that the surface-functionalized $\mathrm{BaTiO}_{3}$ nanoparticles have good compatibility with the polymer matrix. These results are greatly significant for the potential applications of these nanocomposites in film capacitors and embedded capacitors fields.

The temperature-dependence of the dielectric properties of the nanocomposite films was further investigated. The dielectric constant and dielectric loss of the composite film $(20.0 \mathrm{wt} \%)$ were measured as a function of temperature from $25{ }^{\circ} \mathrm{C}$ to $250{ }^{\circ} \mathrm{C}$ at $1 \mathrm{kHz}$, and the results are shown in Fig. 5a. It is clear that the dielectric properties are relatively stable with respect to temperature before $T_{\mathrm{g}}$ and abruptly increase when the temperature is higher than the $T_{\mathrm{g}}$ of PEN. It is because the macromolecular motion is restricted when the temperature is below the $T_{\mathrm{g}}$ of PEN. However, when the temperature is higher than the $T_{\mathrm{g}}$ of PEN, the macromolecular motion and polarization inside the polymer are enhanced. ${ }^{37}$ In addition, the dielectric constant remains almost the same at different temperatures with the increasing frequency (Fig. 5b). These results indicate that the samples have good permittivity-frequency stability even at $160{ }^{\circ} \mathrm{C}$. Furthermore, the breakdown strength of the sample at different temperatures presents a slight decrease lower than that at $160{ }^{\circ} \mathrm{C}$, as shown in Fig. 5c. Therefore, the calculated results indicate that the energy density at different temperatures present a stable trend (Fig. 5d), which also confirm the permittivity-temperature stability of the composite films.

\section{Conclusions}

In summary, novel core-shell-structured CPEN-f-BT@CuPc nanoparticles were successfully prepared by grafting CuPc onto $\mathrm{BaTiO}_{3}$ whose surface was modified by CPEN. Then, the CPEN-f-BT@CuPc/PEN composites films were prepared through a solution casting method combined with an ultrasonic dispersion technology. The results showed that the dielectric constant of the composites at $1 \mathrm{kHz}$ was enhanced from 3.9 to 9.0 and the energy density of the composites increased from 0.70 to $1.13 \mathrm{~J} \mathrm{~cm}^{-3}$ when the content of the nanofillers increased from $0 \%$ to $20.0 \mathrm{wt} \%$, respectively. Furthermore, a stable dielectric constant and energy density were observed for these films in the temperature range of $25-160{ }^{\circ} \mathrm{C}$, which indicates great potential of these nanocomposites for use as organic film capacitors.

\section{Acknowledgements}

The financial support from National Natural Science Foundation of China (Project No. 51603029 and 51373028) and UESTC (Project No. A03013023601012) are gratefully acknowledged.

\section{Notes and references}

1 B. J. Chu, X. Zhou, K. L. Ren, et al., Science, 2006, 313, 334336.

2 A. Imhof and D. J. Pine, Nature, 1997, 389, 948-951.

3 Z. M. Dang, J. K. Yuan, S. H. Yao, et al., Adv. Mater., 2013, 25, 6334-6365.

4 J. Li, S. I. Seok, B. Chu, et al., Adv. Mater., 2009, 21, 217-221.

5 Y. Shen, Y. Lin and Q. M. Zhang, MRS Bull., 2015, 40, 433458.

6 J. N. Coleman, U. Khan and Y. K. Gun'ko, Adv. Mater., 2006, 18, 689-706.

7 J. Yang, H. L. Tang, Y. Q. Zhan, et al., Mater. Lett., 2012, 72, 42-45.

8 Y. You, R. B. Wei, R. Q. Yang, et al., RSC Adv., 2016, 6, 7087770883.

9 R. B. Wei, K. Li, J. Y. Ma, et al., J. Mater. Sci. Mater. Electron., 2016, 27, 9565-9571.

10 Z. J. Pu, L. F. Tong, M. N. Feng, et al., RSC Adv., 2015, 5, 72028-72036.

11 J. Yang, X. L. Yang, Z. J. Pu, et al., Mater. Lett., 2013, 93, 199202.

12 W. Yang, X. L. Yang, Z. J. Pu, et al., J. Appl. Polym. Sci., 2014, 131, 40100-40107.

13 V. K. Thakur and R. K. Gupta, Chem. Rev., 2016, 116, 42604317.

14 D. Padalia, G. Bisht, U. C. Johri, et al., Solid State Sci., 2013, 19, 122-129.

15 X. Zhang, Y. Ma, C. Zhao, et al., Appl. Surf. Sci., 2014, 305, 531-538.

16 L. Xie, X. Huang, C. Wu, et al., J. Mater. Chem., 2011, 21, 5897-5906.

17 T. Hoshina, J. Ceram. Soc. Jpn., 2013, 121, 156-161.

18 C. W. Beier, M. A. Cuevas and R. L. Brutchey, Langmuir, 2010, 26, 5067-5071.

19 X. Huang, M. N. Feng and X. B. Liu, J. Mater. Sci.: Mater. Electron., 2014, 25, 97-102. 
20 L. Xie, X. Huang, Y. Huang, K. Yang and P. Jiang, J. Phys. Chem. C, 2013, 117, 22525-22537.

21 R. X. Wang, Q. Zhu, W. S. Wang, et al., New J. Chem., 2015, 39, 4407-4413.

22 X. Huang, M. N. Feng and X. B. Liu, RSC Adv., 2014, 4, 49854992.

23 K. Yang, X. Huang, Y. Huang, et al., Chem. Mater., 2013, 25, 2327-2338.

24 H. Liu, Y. Shen, Y. Song, et al., Adv. Mater., 2011, 23, 51045108.

25 K. Yu, Y. Niu, Y. Zhou, et al., J. Am. Ceram. Soc., 2013, 96, 2519-2524.

26 H. L. Tang, J. Yang, J. C. Zhong, et al., Mater. Lett., 2011, 65, 1703-1706.

27 Y. You, X. Huang, Z. J. Pu, et al., J. Polym. Res., 2015, 22, 211219.

28 B. Achar and K. J. Lokesh, Organomet. Chem., 2004, 689, 3357-3361.
29 H. L. Tang, P. Wang, P. L. Zheng, et al., Compos. Sci. Technol., 2016, 123, 134-142.

30 Z. C. Wang, W. Yang, J. J. Wei, et al., Mater. Lett., 2014, 123, 6-9.

31 J. H. Zhu, Y. X. Li, Y. Chen, et al., Carbon, 2011, 49, 19001905.

32 W. J. Gammon, O. Kraft, A. C. Reilly, et al., Carbon, 2003, 41, 1917-1923.

33 Z. J. Pu, H. L. Tang, X. Huang, et al., Colloids Surf., A, 2012, 415, 125-133.

34 C. Li, Y. Gu, X. B. Liu, et al., Thin Solid Films, 2006, 515, 18721876.

35 F. He, S. Lau, H. L. Chan, et al., Adv. Mater., 2009, 21, 710715.

36 H. L. Tang, J. C. Zhong, J. Yang, et al., J. Electron. Mater., 2011, 40, 141-148.

37 R. Q. Yang, R. B. Wei, K. Li, et al., Sci. Rep., 2016, 6, 3643436440 . 